\title{
EXTENSIONS OF MCCOY'S THEOREM
}

\author{
CHAN YONG HONG \\ Department of Mathematics and Research Institute for Basic Sciences, \\ Kyung Hee University, Seoul 131-701, Korea \\ e-mail:hcy@khu.ac.kr \\ NAM KYUN KIM* \\ College of Liberal Arts and Sciences, Hanbat National University, \\ Daejeon 305-719, Korea \\ e-mail:nkkim@hanbat.ac.kr \\ and YANG LEE \\ Department of Mathematics Education, Pusan National University, \\ Pusan 609-735, Korea \\ e-mail:ylee@pusan.ac.kr
}

(Received 24 August 2007; accepted 28 July 2009)

\begin{abstract}
McCoy proved that for a right ideal $A$ of $S=R\left[x_{1}, \ldots, x_{k}\right]$ over a ring $R$, if $r_{S}(A) \neq 0$ then $r_{R}(A) \neq 0$. We extend the result to the Ore extensions, the skew monoid rings and the skew power series rings over non-commutative rings and so on.

2000 MSC: Primary: 16S36, 16N60; Secondary: 13B25, 16W20.
\end{abstract}

Over a commutative ring $R$, McCoy [4, Theorem 2] obtained the following in 1942: $f(x)$ is a zero divisor in $R[x]$ if and only if $f(x) c=0$ for some non-zero $c \in R$, where $R[x]$ is the polynomial ring with indeterminate $x$ over $R$. But Weiner [9] showed that this theorem fails in non-commutative rings.

Based on these results, Nielsen [6] called a ring $R$ right McCoy when the equation $f(x) g(x)=0$ implies $f(x) c=0$ for some non-zero $c \in R$, where $f(x), g(x)$ are non-zero polynomials in $R[x]$. Left McCoy rings are defined similarly. If a ring is both left and right McCoy then the ring is called a McCoy ring. Nielsen [6, Theorem 2] proved that if a ring $R$ is reversible (i.e. for $a, b \in R, a b=0$ implies $b a=0$ ) then $R$ is McCoy.

As stated above, McCoy's theorem fails in non-commutative rings. However McCoy [5] proved the following result.

THEOREM $\dagger$. Let $R$ be a ring and $A$ a right ideal of $S=R\left[x_{1}, \ldots, x_{k}\right]$. If $r_{S}(A) \neq 0$ then $r_{R}(A) \neq 0$.

In 2002, Hirano [3, Theorem 2.2] proved independently that if for $f(x) \in R[x]$, $r_{R[x]}(f(x) R[x]) \neq 0$ then $r_{R}(f(x) R[x]) \neq 0$.

On the other hand, McCoy's theorem fails in the formal power series ring $R[[x]]$ over a commutative ring $R$ by [1, Example 3] in general. However, Gilmer [2] provided several conditions that are sufficient in order that the analogue of McCoy's theorem should be valid in a commutative $R[[x]]$. Such conditions include the reducedness

* Corresponding author 
and the von Neumann regularity of the total quotient ring, etc. Moreover, Fields [1, Theorem 5] proved that if $R$ is a commutative Noetherian ring in which $Q_{1} \cap Q_{2} \cap \cdots \cap$ $Q_{n}=0$ is a shortest primary representation of 0 , then $f(x) g(x)=0$ implies $f(x) c=0$ for some non-zero $c \in R$.

We extend, in this paper, Theorem $\dagger$ to the Ore extensions of several types, the skew monoid rings and the skew power series rings over non-commutative rings, and so on.

Throughout this paper, $R$ denotes associative ring with identity. We denote the right annihilator of $A$ in $R$ by $r_{R}(A)$, where $A$ is a subset of an extension of $R$. We assume that $\sigma$ is an automorphism of $R$ and $\delta$ is a $\sigma$-derivation of $R$. Recall that the Ore extension $R[x ; \sigma, \delta]$ of a ring $R$ is the ring obtained by giving the polynomial ring over $R$ with the new multiplication $x r=\sigma(r) x+\delta(r)$ for any $r \in R$.

THEOREM 1. Let $R$ be a ring and and $A$ a right ideal of $S=R[x ; \sigma, \delta]$. If $r_{S}(A) \neq 0$ then $r_{R}(A) \neq 0$.

Proof. Let $g(x)=b_{0}+b_{1} x+\cdots+b_{n} x^{n}$ be a non-zero element in $r_{S}(A)$ with minimal degree. Then $\operatorname{Ag}(x)=0$ and so $f(x) \operatorname{Sg}(x)=0$ for any $f(x)=a_{0}+a_{1} x+\cdots+$ $a_{m} x^{m} \in A$. Note that for any $r \in R$,

$$
\begin{aligned}
r x^{i}= & x^{i} \sigma^{-i}(r)-\left(\sum_{s+t=i-1} \sigma^{s} \delta \sigma^{t}\left(\sigma^{-i}(r)\right)\right) x^{i-1} \\
& -\cdots-\left(\sum_{s+t=i-1} \delta^{s} \sigma \delta^{t}\left(\sigma^{-i}(r)\right)\right) x-\delta^{i}\left(\sigma^{-i}(r)\right) .
\end{aligned}
$$

Then we can rewrite $f(x)=c_{0}+x c_{1}+\cdots+x^{m} c_{m}$. Thus we have the following:

$$
\left(c_{0}+x c_{1}+\cdots+x^{m} c_{m}\right) R\left(b_{0}+b_{1} x+\cdots+b_{n} x^{n}\right)=0 .
$$

We will show that $f(x) b_{j}=0$ for any $0 \leq j \leq n$. If $n=0$, then we are done. Suppose that $n \geq 1$. From equation $(*)$, we have $c_{m} b_{n}=0$. Then $f(x) R\left(c_{m} g(x)\right) \subseteq f(x) R g(x)=0$ and so equation $(*)$ becomes

$$
\left(c_{0}+x c_{1}+\cdots+x^{m} c_{m}\right) R\left(c_{m} b_{0}+c_{m} b_{1} x+\cdots+c_{m} b_{n-1} x^{n-1}\right)=0 .
$$

By the choice of $g(x)$, we have $c_{m} b_{0}+c_{m} b_{1} x+\cdots+c_{m} b_{n-1} x^{n-1}=0$ and so $c_{m} b_{j}=0$ for any $0 \leq j \leq n$. Assume that $c_{i} b_{j}=0$, where $i=t+1, \ldots, m$ and $0 \leq j \leq n$ and that for each $0 \leq i \leq t, c_{i} b_{j} \neq 0$ for some $j$. Then equation $(*)$ becomes

$$
0=f(x) R g(x)=\left(c_{0}+x c_{1}+\cdots+x^{t} c_{t}\right) R\left(b_{0}+b_{1} x+\cdots+b_{n} x^{n}\right) .
$$

Thus we also have $c_{t} b_{n}=0$. Then $f(x) R\left(c_{t} g(x)\right) \subseteq f(x) R g(x)=0$ and so $f(x) R\left(c_{t} b_{0}+\right.$ $\left.c_{t} b_{1} x+\cdots+c_{t} b_{n-1} x^{n-1}\right)=0$. By the choice of $g(x)$, we have $c_{t} b_{0}+c_{t} b_{1} x+\cdots+$ $c_{t} b_{n-1} x^{n-1}=0$ and so $c_{t} b_{j}=0$ for any $0 \leq j \leq n$, which is a contradiction. Consequently $n$ must be zero. Hence $f(x) b_{0}=0$ and therefore $A b_{0}=0$ with $b_{0} \neq 0$.

COROLlary 2. For a ring $R$, let $T$ be $R[x ; \sigma], R\left[x, x^{-1} ; \sigma\right]$ or $R[x ; \delta]$ and $A$ a right ideal of $T$. If $r_{T}(A) \neq 0$ then $r_{R}(A) \neq 0$. 
Recall that a monoid $G$ is called a unique product monoid (simply, u.p.-monoid) if any two non-empty finite subsets $A, B \subseteq G$ there exists $c \in G$ uniquely presented in the form $a b$ where $a \in A$ and $b \in B$. The class of u.p.-monoids is quite large and important (see [7] and [8] for details). For example, this class includes the right or left ordered monoids, submonoids of a free group, and torsion-free nilpotent groups.

Let $R$ be a ring and $G$ a u.p.-monoid. Assume that $G$ acts on $R$ by means of a homomorphism into the automorphism group of $R$. We denote by $\sigma_{g}(r)$ the image of $r \in R$ under $g \in G$. The skew monoid ring $R * G$ is a ring which as a left $R$-module is free with basis $G$ and multiplication defined by the rule $g r=\sigma_{g}(r) g$.

THEOREM 3. Let $R$ be a ring, $G$ a u.p.-monoid and $A$ a right ideal of $R * G$. If $r_{R * G}(A) \neq 0$ then $r_{R}(A) \neq 0$.

Proof. Let $\beta=b_{0} h_{0}+b_{1} h_{1}+\cdots+b_{n} h_{n}$ be a non-zero element in $r_{R * G}(A)$ with minimal non-zero terms, where $b_{j} \in R$ and $h_{j} \in G$. Then $A \beta=0$ and so $\alpha(R * G) \beta=0$ for any $\alpha=a_{0} g_{0}+a_{1} g_{1}+\cdots+a_{m} g_{m} \in A$ with $a_{i} \in R$ and $g_{i} \in G$. Thus we have the following:

$$
\left(a_{0} g_{0}+a_{1} g_{1}+\cdots+a_{m} g_{m}\right) R\left(b_{0} h_{0}+b_{1} h_{1}+\cdots+b_{n} h_{n}\right)=0 .
$$

We will show that $a_{i} R \sigma_{g_{i}}\left(b_{j}\right)=0$ for any $0 \leq i \leq m$ and $0 \leq j \leq n$. If $n=0$, then

$$
\begin{aligned}
0 & =\left(a_{0} g_{0}+a_{1} g_{1}+\cdots+a_{m} g_{m}\right) r\left(b_{0} h_{0}\right) \\
& =a_{0} \sigma_{g_{0}}\left(r b_{0}\right) g_{0} h_{0}+a_{1} \sigma_{g_{1}}\left(r b_{0}\right) g_{1} h_{0}+\cdots+a_{m} \sigma_{g_{m}}\left(r b_{0}\right) g_{m} h_{0} .
\end{aligned}
$$

By [7, Lemma 1, p.119], $g_{i} h_{0} \neq g_{j} h_{0}$ if $i \neq j$. Thus $a_{i} R \sigma_{g_{i}}\left(b_{0}\right)=0$. Suppose that $n \geq$ 1. Since $G$ is a u.p.-monoid, there exist $g_{p}, h_{q}$ such that $g_{p} h_{q}$ is uniquely presented by considering two subsets $A=\left\{g_{0}, g_{1}, \ldots, g_{m}\right\}$ and $B=\left\{h_{0}, h_{1}, \ldots, h_{n}\right\}$ of $G$. After reordering if necessary, we may assume that $p=m$ and $q=n$. Then from equation $(* *)$, we have $a_{m} R \sigma_{g_{m}}\left(b_{n}\right)=0$. Since $\sigma_{g_{m}}$ is an automorphism of $R, \sigma_{g_{m}}^{-1}\left(a_{m}\right) R b_{n}=$ 0 . Now for any $s \in R, \alpha R\left(\sigma_{g_{m}}^{-1}\left(a_{m}\right) s \beta\right) \subseteq \alpha R \beta=0$ and so $\alpha R\left(\sigma_{g_{m}}^{-1}\left(a_{m}\right) s \beta\right)=0$, where $\sigma_{g_{m}}^{-1}\left(a_{m}\right) s \beta=\sigma_{g_{m}}^{-1}\left(a_{m}\right) s b_{0} h_{0}+\sigma_{g_{m}}^{-1}\left(a_{m}\right) s b_{1} h_{1}+\cdots+\sigma_{g_{m}}^{-1}\left(a_{m}\right) s b_{n-1} h_{n-1}$. By the choice of $\beta, \sigma_{g_{m}}^{-1}\left(a_{m}\right) s \beta=0$, and hence $a_{m} R \sigma_{g_{m}}\left(b_{j}\right)=0$ for any $0 \leq j \leq n$. After reordering if necessary, assume that $a_{i} R \sigma_{g_{i}}\left(b_{j}\right)=0$, where $i=t+1, \ldots, m$ and $0 \leq j \leq n$ and that for each $0 \leq i \leq t, a_{i} R \sigma_{g_{i}}\left(b_{j}\right) \neq 0$ for some $j$. Then from equation (**), we have $\alpha R \beta=$ $\left(a_{0} g_{0}+a_{1} g_{1}+\cdots+a_{t} g_{t}\right) R\left(b_{0} h_{0}+b_{1} h_{1}+\cdots+b_{n} h_{n}\right)=0$. Since $G$ is an u.p.-monoid, there exist $p, q$ with $0 \leq p \leq t$ and $0 \leq q \leq n$ such that $g_{p} h_{q}$ is uniquely presented by considering two subsets $A=\left\{g_{0}, g_{1}, \ldots, g_{t}\right\}$ and $B=\left\{h_{0}, h_{1}, \ldots, h_{n}\right\}$ of $G$. After reordering if necessary, we may assume that $p=t$ and $q=n$. Then $a_{t} R \sigma_{g_{t}}\left(b_{n}\right)=0$ and so $\sigma_{g_{t}}^{-1}\left(a_{t}\right) R b_{n}=0$. Hence

$$
\begin{aligned}
0 & =\alpha R\left(\sigma_{g_{t}}^{-1}\left(a_{t}\right) s \beta\right) \\
& =\alpha R\left(\sigma_{g_{t}}^{-1}\left(a_{t}\right) s b_{0} h_{0}+\sigma_{g_{t}}^{-1}\left(a_{t}\right) s b_{1} h_{1}+\cdots+\sigma_{g_{t}}^{-1}\left(a_{t}\right) s b_{n-1} h_{n-1}\right) .
\end{aligned}
$$

By choice of $\beta$, we have $\sigma_{g_{t}}^{-1}\left(a_{t}\right) s b_{0} h_{0}+\sigma_{g_{t}}^{-1}\left(a_{t}\right) s b_{1} h_{1}+\cdots+\sigma_{g_{t}}^{-1}\left(a_{t}\right) s b_{n-1} h_{n-1}=0$ and hence $a_{t} R \sigma_{g_{t}}\left(b_{j}\right)=0$ for any $0 \leq j \leq n$, which is a contradiction. Consequently $n$ must be zero. Hence we have $\alpha b_{0}=0$, and therefore $A b_{0}=0$ with $b_{0} \neq 0$.

By [1, Example 3], McCoy's theorem fails in the formal power series ring $R[[x]]$ over a commutative ring $R$. However, Gilmer [2] proved that a commutative ring satisfies 
McCoy's theorem for the formal power series ring case, when it is reduced (i.e. a ring with no non-zero nilpotent elements).

We here show that Theorem $\dagger$ holds for the skew power series rings and the skew Laurent power series rings over semi-prime rings, noting that Theorem $\dagger$ does not hold for the formal power series ring case in general.

Lemma 4. Let $R$ be a semi-prime ring. Then for $f(x)=\sum_{i=0}^{\infty} a_{i} x^{i}, g(x)=\sum_{j=0}^{\infty} b_{j} x^{j} \in$ $R[[x ; \sigma]], f(x) R[[x ; \sigma]] g(x)=0$ if and only if $a_{i} R \sigma^{i+t}\left(b_{j}\right)=0$ for all $t, i, j \geq 0$.

Proof. It is enough to show the necessity. Suppose that $f(x) R[[x ; \sigma]] g(x)=0$, equivalently, $f(x) x^{t} \operatorname{rg}(x)=0$ for any $r \in R$ and integer $t \geq 0$. So we have the following:

$$
\begin{aligned}
a_{0} \sigma^{t}\left(r b_{0}\right) & =0, \\
a_{0} \sigma^{t}\left(r b_{1}\right)+a_{1} \sigma^{t+1}\left(r b_{0}\right) & =0, \\
\cdots & \\
a_{0} \sigma^{t}\left(r b_{n}\right)+a_{1} \sigma^{t+1}\left(r b_{n-1}\right)+\cdots+a_{n} \sigma^{t+n}\left(r b_{0}\right) & =0 .
\end{aligned}
$$

From equation (0), $a_{0} \sigma^{t}\left(r b_{0}\right)=0$. In equation (1), we replace $r$ by $r b_{0} s$ for any $s \in R$. Then $0=a_{0} \sigma^{t}\left(r b_{0} s b_{1}\right)+a_{1} \sigma^{1+t}\left(r b_{0} s b_{0}\right)=a_{1} \sigma^{1+t}\left(r b_{0} s b_{0}\right)$. Thus $a_{1} R \sigma^{1+t}\left(b_{0}\right) R \sigma^{1+t}\left(b_{0}\right)=0$. Since $R$ is semi-prime, $a_{1} R \sigma^{1+t}\left(r b_{0}\right)=0$ and so $a_{1} \sigma^{1+t}\left(r b_{0}\right)=0$ for all $r \in R$. From equation (1), $a_{0} \sigma^{t}\left(r b_{1}\right)=0$ for all $r \in R$. Now suppose that $a_{i} \sigma^{i+t}\left(r b_{j}\right)=0$ for all $t \geq 0$ and $0 \leq i+j \leq n-1$. In equation $(n)$, we first replace $r$ by $r b_{0} s$. Then $a_{n} \sigma^{n+t}\left(r b_{0} s b_{0}\right)=0$ and so $a_{n} \sigma^{n+t}\left(r b_{0}\right)=0$ by the same method as above. So we have

$$
a_{0} \sigma^{t}\left(r b_{n}\right)+a_{1} \sigma^{1+t}\left(r b_{n-1}\right)+\cdots+a_{n-1} \sigma^{n-1+t}\left(r b_{1}\right)=0 .
$$

Next, we replace $r$ by $r b_{1} s$ for any $s \in R$ in equation $\left(n^{\prime}\right)$. Then $a_{n-1} \sigma^{n-1+t}\left(r b_{1}\right)=0$ using $R$ is semi-prime. Continuing this process, we have $a_{i} \sigma^{i+t}\left(r b_{j}\right)=0$ for all $t \geq 0$ and $0 \leq i+j \leq n$. By induction, we have $a_{i} \sigma^{i+t}\left(r b_{j}\right)=0$ and therefore $a_{i} R \sigma^{i+t}\left(b_{j}\right)=0$ for all $k, i, j \geq 0$.

We also have the same result as Lemma 4 for the skew Laurent power series ring $R\left[\left[x, x^{-1} ; \sigma\right]\right]$, using a slightly modified method. Now we have the following.

THEOREM 5. Let $R$ be a semi-prime ring and $A$ a right ideal of $T=R[[x ; \sigma]]$ or $T=R\left[\left[x, x^{-1} ; \sigma\right]\right]$. If $r_{T}(A) \neq 0$ then $r_{R}(A) \neq 0$.

Proof. It is enough to show the skew power series ring case. Let $0 \neq g(x)=$ $\sum_{j=0}^{\infty} b_{j} x^{j} \in r_{T}(A)$. Then $A g(x)=0$ and so $f(x) T g(x)=0$ for any $f(x)=\sum_{i=0}^{\infty} a_{i} x^{i} \in A$. By Lemma 4, we have $a_{i} R \sigma^{i+t}\left(b_{j}\right)=0$ for any integers $t, i, j \geq 0$. Then $f(x) b_{j}=0$ and therefore $A c=0$, where $c=b_{j}$ for any non-zero $b_{j}$.

ACKNOWLEDGEMENTS. The first named author was supported by the Kyung Hee University in 2007, the second named author was supported by the National Research Foundation of Korea grant funded by the Korea Government (No. 2009-0074018), while the third named author was supported by the Korea Research Foundation Grant funded by the Korea Government (KRF-2008-521-C00003). 


\section{REFERENCES}

1. D. E. Fields, Zero divisors and nilpotent elements in power series rings, Proc. Amer. Math. Soc. 27 (1971), 427-433.

2. R. Gilmer and T. Parker, Zero divisors in power series rings, J. Reine Angew. Math. 278/279 (1975), 145-164.

3. Y. Hirano, On annihilator ideals of a polynomial ring over a noncommutative ring, $J$. Pure Appl. Algebra 168 (2002), 45-52.

4. N. H. McCoy, Remarks on divisors of zero, Amer. Math. Monthly 49 (1942), 286-295.

5. N. H. McCoy, Annihilators in polynomial rings, Amer. Math. Monthly 64 (1957), 28-29. 134-141.

6. P. P. Nielsen, Semi-commutativity and the McCoy condition, J. Algebra 298 (2006),

7. J. Okninski, Semigroup algebras (Marcel Dekker, New York, 1991).

8. D. S. Passmann, The algebraic structure of group rings (John Wiley \& Sons, New York, 1977).

9. L. Weiner, Concerning a theorem of McCoy, Amer. Math. Monthly 59 (1952), 336-337. 